\title{
Alocação de aeronaves a voos considerando restrições operacionais, de manutenção e de desempenho das aeronaves
}

\author{
João Carlos Medau ${ }^{1}$, Nicolau D. F. Gualda ${ }^{2}$
}

${ }^{1}$ Departamento de Engenharia de Transportes, Escola Politécnica, Universidade de São Paulo, medau@gmail.com 2Departamento de Engenharia de Transportes, Escola Politécnica, Universidade de São Paulo, ngualda@usp.br

\section{Recebido: \\ 5 de fevereiro de 2017 \\ Aceito para publicação: \\ 22 de maio de 2018 \\ Publicado: \\ 31 de agosto de 2018 \\ Editor de área: \\ Li Weigang}

\section{Palavras-chaves:}

Alocação de aeronaves e voos, Heurística,

Programação linear inteira,

Lista tabu.

\section{Keywords:}

Tail assignment problem (TAP), Integer linear programming, Heuristic,

Tabu list,

DOI:10.14295/transportes.v25i2.1316

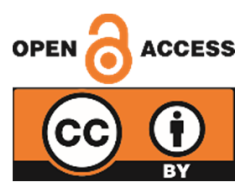

\begin{abstract}
RESUMO
Este trabalho apresenta um modelo matemático exato, baseado em programação linear inteira, e um método heurístico, baseado na meta-heurística Busca Tabu, para a solução do problema de alocação de aeronaves a voos, ou tail assignment problem (TAP), considerando as restrições de conectividade de voos, permanência de aeronaves no solo, serviços de manutenção, limitações técnicas de aeronaves, conexões de passageiros e tripulantes e desempenho de aeronaves, visando a minimizar o custo total da operação. Os modelos desenvolvidos foram aplicados a problemas reais, extraídos da malha de uma empresa brasileira e os resultados obtidos apresentam ganhos em relação ao método de alocação de aeronaves utilizado na operação diária. Os tempos computacionais para solução pelo método exato são longos, indicando que o método heurístico é mais adequado, com resultados de boa qualidade obtidos em tempos computacionais razoáveis aceitáveis.
\end{abstract}

\section{ABSTRACT}

This paper presents and integer programming mathematical model and a heuristic method based on the Tabu Search meta-heuristic to solve the tail assignment problem (TAP), considering flight connections restrictions, ground connection times, maintenance services, aircraft technical limitations and aircraft performance, aiming to minimize the total operation costs. The models were used to solve real problems, extracted from the network of a Brazilian airline and the results show savings when compared with the traditional aircraft allocation method used by the airline. The MIP computer running times are long, indicating the heuristic method is more suitable for airline operations, giving good quality results in reasonable running times.

\section{INTRODUÇÃO}

Com os altos custos operacionais (MARTIN, 2011), as empresas aéreas têm grande dificuldade em obter e manter lucratividade atrativa para seus investidores. Estudos mostram que grandes empresas aéreas podem ter lucros muito baixos, da ordem de apenas $1 \%$ do faturamento (Mc Cartney, 2012). Face à baixa lucratividade e às dificuldades para aumentá-la variando-se as tarifas (BELOBABA et al., 2009), processos de otimização ganham importância nas operações de empresas aéreas, de forma a diminuir custos, tanto quanto possível, sem prejudicar a qualidade dos serviços oferecidos aos clientes.

Modelos de otimização estão presentes em diversas etapas do planejamento e execução das operações de empresas aéreas, desde a determinação das rotas a serem voadas (CAETANO, 
2011), cálculo de tarifas (MCAFEE e VELDE, 2007), definição da frota para cada rota (CAETANO e GUALDA 2011, 2015), alocação de aeronaves (GRÖNKVIST, 2005; BAZARGAN, 2010; LAPP e WIKENHAUSER, 2012; GONZÁLEZ, 2014), programação de tripulantes (GOMES e GUALDA 2011, 2015), até o cálculo da quantidade ideal de combustível para cada voo (FREGNANI, 2007). Trabalhos da literatura apontam ganhos da ordem de milhões de dólares por ano em grandes empresas que empregam otimização na solução dos problemas de alocação de frotas (SUBRAMANIAN et al, 1999) e de aeronaves, destacando a importância de tais técnicas em suas operações.

O objetivo deste trabalho é apresentar um modelo matemático exato e um método heurístico de alocação de aeronaves a voos, considerando as diversas restrições operacionais, de condições técnicas e desempenho das aeronaves, de manutenção e de eventuais restrições de aeroportos, bem como preferências operacionais das empresas aéreas que, normalmente, não fazem parte de modelos de otimização. Tais preferências são importantes, pois nascem da observação diária das operações pelo pessoal das empresas, que precisam observar restrições em função de características peculiares do cenário brasileiro. Quando tais preferências não são incluídas nos modelos de otimização, o que se nota é que o pessoal responsável pela operação passa a ignorar os resultados das otimizações, usando muitas vezes soluções manuais, que demandam maior tempo para ser produzidas e não garantem redução de custos. Neste sentido, a principal contribuição deste trabalho é considerar uma ampla gama de restrições operacionais existentes no dia-a-dia das empresas aéreas, possibilitando o uso dos modelos propostos em problemas reais, com ganhos na eficiência operacional.

0 trabalho está estruturado da seguinte forma: na seção 2 o TAP é descrito em detalhes, com as diversas restrições consideradas na modelagem matemática e no desenvolvimento do método heurístico; na seção 3 são apresentadas abordagens ao TAP encontradas na literatura; na seção 4 é apresentado o modelo matemático exato desenvolvido; na seção 5 é apresentado o desenvolvimento do método heurístico; na seção 6 é apresentada a aplicação dos métodos exato e heurístico à malha de uma empresa aérea brasileira de médio porte e os resultados obtidos; na seção 7 é apresentada a conclusão do trabalho e as possibilidades de continuidade da pesquisa.

\section{DEFINIÇÃO DO PROBLEMA DE ALOCAÇÃO DE AERONAVES}

Dada a malha de uma empresa aérea e sua divisão entre cada um dos tipos de aeronave existentes na frota, cada voo deve ser alocado a uma aeronave específica do tipo correspondente, resolvendo o problema conhecido como problema de alocação de aeronaves, aircraft assignment problem, aircraft routing problem ou tail assignment problem (CLARKE et al, 1997). A sequência de voos realizada por uma certa aeronave é conhecida como trilho e, em diversas empresas, é tratado apenas como um problema de viabilidade, onde devem ser garantidas a realização de todos os voos e dos serviços de manutenção mais simples, sem preocupações com economia (KLABDJAN, 2004). As malhas de voos das empresas obedecem a ciclos que se repetem, usualmente, em intervalos de um dia para voos domésticos e de uma semana para voos internacionais (BAZARGAN, 2010).

A primeira restrição a ser atendida consiste em considerar que uma aeronave candidata à realização de um voo deve estar posicionada no aeroporto de origem com tempo suficiente para sua realização, incluindo o tempo necessário para os procedimentos de preparação para o voo, 
abastecimento, embarque dos passageiros, etc. Além dos tempos mínimos de trânsito necessários para a preparação da aeronave, em determinados voos e aeroportos, são necessários tempos maiores, especialmente nos grandes $h u b s$, onde os passageiros precisam desembarcar, passar por procedimentos de segurança, imigração e alfândega até chegar às salas de embarque dos voos de conexão. Nestes casos, o modelo deve considerar restrições de tempo de solo específicas por aeroporto e por voo, caso existam.

A empresa operadora da aeronave deve pagar ao administrador do aeroporto uma tarifa referente ao pouso (taxa de pouso) e, quando uma aeronave permanece no pátio de estacionamento de um aeroporto aguardando para realizar o próximo voo, deve pagar também uma taxa ao tempo em que a aeronave permanece estacionada (taxa de permanência). Tais tarifas varia de acordo com a categoria do aeroporto e o peso máximo de decolagem das aeronaves. (GRU AIRPORT, 2014; INFRAERO, 2014; INFRAMERICA, 2014). As tarifas de navegação não são consideradas no modelo apresentado neste trabalho, pois, para aeronaves da mesma família (mesma faixa de peso), as tarifas são iguais (DECEA, 2015).

Dois tipos básicos de problemas são normalmente enfrentados pelas empresas aéreas e devem ser resolvidos pelos modelos que solucionam o TAP:

- Problema de planejamento de frota no cenário estratégico, onde, dados a malha atual, voos que se pretende incluir e/ou excluir da malha e respectivas demandas, deseja-se saber qual a quantidade de cada tipo de aeronave é necessária para realizar-se tal malha;

- Problema de alocação de aeronaves à malha atual da empresa, resolvidos nos cenários tático e operacional. Neste caso, a malha já definida da empresa é alocada às aeronaves da frota já existente para a realização dos voos, de acordo com as restrições operacionais.

Alguns custos de operação das aeronaves são relacionados à quantidade de horas de voo realizada num período, e podem variar de uma aeronave para outra. Um exemplo típico é o custo de leasing dos motores que, muitas vezes, não está incluído no valor do leasing pago mensalmente pelo restante da aeronave (chamado airframe). 0 leasing dos motores depende essencialmente da tração média usada nas decolagens realizadas por cada uma das aeronaves e, quanto maior a tração média utilizada, maior o preço do leasing por hora voada (ACKERT, 2011).

Com o uso, as aeronaves ficam cada vez menos eficientes, com diminuição de seu alcance específico, isto é, consomem mais combustível para voar uma certa distância. Este aumento de consumo tem basicamente duas razões: diminuição da eficiência dos motores, que passam a consumir mais combustível para gerar a mesma tração e aumento do arrasto parasita da aeronave, causado por superfícies de comando desajustadas, selos de portas com vazamentos e reparos estruturais (AIRBUS, 2002). A diferença entre o desempenho de uma aeronave de referência e o que realmente ocorre, conhecido como fator de degradação, é utilizado para inserir correções de consumo nos planos de voo e computadores de bordo. Tratando-se de um valor percentual calculado para cada aeronave da frota, fica claro que as aeronaves têm consumos diferentes entre si na realização dos voos e, caso o TAP seja resolvido de forma a alocar as aeronaves com maior degradação aos voos mais curtos, haverá uma economia global de combustível pela empresa (LAPP e WIKENHAUSER, 2012).

Para que uma aeronave seja considerada aeronavegável e possa realizar um voo, todas as atividades previstas no programa de manutenção devem ter sido realizadas e estar dentro do seu prazo de validade. As inspeções de linha, especialmente as realizadas diária e semanalmente, por sua característica repetitiva no curto prazo, devem ser tratadas pelo modelo de solução do TAP como atividades que devem ser programadas como parte da solução do problema. 
Assim, o modelo deve respeitar o tempo mínimo de execução de cada inspeção, o intervalo máximo entre execuções consecutivas de um mesmo tipo de inspeção e os aeroportos onde a empresa é certificada para realizar as tarefas, bem como os custos inerentes a tal execução.

Alguns tipos de aeronaves pertencem a famílias criadas pelos fabricantes, de forma que têm programas de manutenção, operação e tripulações iguais, apesar de possuírem algumas características diferentes. Um exemplo de família são as aeronaves Airbus A318, A319, A320 e A321. Outro exemplo de família de aeronaves é o Boeing 737, com versões que vão desde 120 até 220 passageiros (BOEING, 2015) e também contam com tripulações, programa de manutenção e componentes em comum.

No caso de empresas que utilizam mais de um tipo de aeronave da mesma família, o TAP pode estar voltado para resolver o subproblema de cada tipo de aeronave individualmente, mas em casos de contingência, onde a empresa tenha problemas de disponibilidade de aeronaves de um determinado tipo, o TAP pode ser resolvido para a família toda, realizando a cobertura de todos os voos da malha, mas com trocas de tipos de aeronaves em relação aos originalmente previstos. Tais trocas podem causar sobra de passageiros (denied boarding ou overbooking) em alguns voos, caso seja utilizada uma aeronave de menor capacidade do que a originalmente programada, ou sobra de lugares vazios (spoilage), caso seja utilizada uma aeronave maior do que a originalmente programada (BELOBABA et al, 2009). Em qualquer um dos dois casos, o modelo deve levar em consideração o custo extra causado por essa diferença, além da diferença de custo operacional por hora de voo entre os diferentes tipos.

Em condições normais de utilização da frota, as aeronaves devem realizar uma quantidade de horas de voo e pousos próximas umas das outras. Já em condições específicas, decorrentes do planejamento de manutenção, por exemplo, uma aeronave poderá ter uma quantidade limitada de horas e/ou pousos disponíveis ou então, preferivelmente voar uma quantidade grande de horas de voo até a data de uma certa inspeção de manutenção já programada (SRIRAM e HAGHANI, 2003). Para que cada aeronave realize a quantidade de horas de voo e pousos mais próximas possível da quantidade ideal, a diferença entre as horas e pousos atribuídos a cada uma, nas soluções geradas pelo modelo matemático, e a quantidade ideal, deve ser penalizada pelo modelo matemático.

Em malhas do tipo hub and spoke, onde muitas conexões ocorrem simultaneamente no hub, as empresas aéreas preferem que voos com grande número de passageiros em conexão sejam executados pela mesma aeronave, minimizando a quantidade de pessoas que precisam desembarcar e reembarcar. A permanência a bordo de passageiros minimiza os tempos de trânsito e aumenta a qualidade do serviço oferecido aos clientes, já que estes preferem permanecer a bordo ao invés de desembarcar e reembarcar em outra aeronave (SIMPSON e BELOBABA, 1992; JARRAH e STREHLER, 2000).

Dentro de uma subfrota de aeronaves de um mesmo tipo, podem ocorrer variações que impeçam ou prejudiquem a operação de uma aeronave específica em um determinado aeroporto e/ou rota. Exemplos comuns de tais variações são: diferentes pesos máximos de decolagem e pouso; ausência de determinados equipamentos e instrumentos de navegação; configuração de tanques de combustível, entre outras. Por exemplo: aeronaves que possuem tanques de combustível com capacidade menor não podem realizar rotas mais longas; aeronaves não equipadas com GPS não podem operar em aeroportos onde este é necessário para realizar aproximações por instrumentos. Além disso, ainda que as aeronaves possuam configurações iguais, algumas podem temporariamente voar com certos equipamentos inoperantes, até que o problema seja 
corrigido. Estes desvios da configuração original são aprovados pelas autoridades aeronáuticas por meio de um documento chamado Lista de Equipamentos Mínimos (AIRBUS, 2005).

Apesar dos voos serem permitidos em tais condições, podem ocorrer limitações operacionais mais severas ou ainda necessidade de equipamentos de apoio de solo nos aeroportos envolvidos (exemplos: menor peso máximo de pouso em caso de conjunto de freio inoperante; necessidade de fontes elétrica e pneumática externas em caso de APU inoperante). Nestes casos, o modelo deve levar tais restrições em consideração e não permitir a alocação de aeronaves com limitações operacionais a voos que operem em aeroportos e/ou trechos que não possam ser atendidos com aeronaves limitadas.

Usualmente, os trilhos das aeronaves gerados na solução do TAP são usados como dado de entrada para a solução do problema de programação de tripulantes, fazendo com que as viagens das tripulações acompanhem, tanto quanto possível, os trilhos das aeronaves (SOARES, 2007). No entanto, quando é necessário realizar alguma mudança no cenário operacional, com trocas não previstas de aeronaves, pode ser interessante alocar uma determinada aeronave numa viagem já predefinida para uma certa tripulação. Essa possibilidade é importante, por exemplo, quando é necessário minimizar atrasos na malha da empresa. Sendo assim, o modelo matemático de solução do TAP deve prever a possibilidade de penalizar soluções que obrigarão a trocas de tripulações.

\section{O PROBLEMA NA LITERATURA}

Grönkvist (2005) apresenta um modelo que considera restrições operacionais, de aeroportos, manutenção e atividades pré-atribuídas. A solução apresentada é baseada em programação matemática e geração de colunas. 0 modelo foi aplicado com sucesso em problemas com até 33 aeronaves, o que corresponde à frota de uma empresa aérea de médio porte. 0 modelo tem grande flexibilidade, especialmente por considerar um custo diferente para cada conexão entre dois nós da rede para cada aeronave, mas transfere parte do problema para uma fase anterior, já que todos os custos que o usuário queira incluir no modelo devem ser previamente computados. Além disso, a formulação apresentada dificulta a atribuição dos custos de manutenção específicos de cada nó candidato e não prevê a inclusão de limitação da capacidade de manutenção de cada base. A utilização de aeronaves fictícias é dificultada pela necessidade de inserção dos custos já unificados, sendo necessário uma etapa de pós-processamento para identificar quantas aeronaves foram utilizadas. Finalmente, o autor não menciona a possibilidade de utilização do modelo para solucionar problemas com famílias de aeronaves, penalizando a utilização de modelos diferentes do inicialmente previsto para cada voo.

Bazargan (2010) apresenta um modelo simples para solução do TAP, baseado no trabalho de Kabbani (1992). 0 modelo baseia-se numa etapa preliminar, onde todas as combinações possíveis de voos para uma aeronave são geradas e o respectivo custo de cada combinação calculado. Tal custo é, na verdade, uma composição de pseudocustos atribuídos pela empresa aérea, de forma a tornar menos atrativas combinações desfavoráveis, como aquelas com curtos tempos de conexão ou rotas circulares, onde uma aeronave fica voando apenas entre um pequeno número de aeroportos. 0 modelo não leva em consideração custos de manutenção, degradação de desempenho das aeronaves, restrições de aeroportos, de conexão de passageiros e não pode ser utilizado para famílias de aeronaves.

Lapp e Wikenhauser (2012) apresentam uma proposta de solução que visa a minimizar o 
consumo de combustível total da empresa, alocando aeronaves com menor degradação aos maiores voos. No entanto, o modelo apresentado não contempla a alocação de tarefas de manutenção, que devem ser atribuídas previamente, nem restrições quanto a aeroportos, conexões de passageiros, custos operacionais (além do combustível) das aeronaves, etc. 0 modelo apresentado é baseado exclusivamente nos custos de combustível e degradação de desempenho das aeronaves, não levando em consideração restrições de manutenção, de aeroportos e conexão de passageiros e não pode ser usado para famílias de aeronaves.

González (2014) apresenta um modelo para solução integrada dos problemas de fleet assignment, aircraft routing e crew pairing para um consórcio de três empresas regionais, cuja característica principal é operar uma malha de cerca de 150 voos diários apenas no período diurno, não havendo operações entre 11:00 da noite e 07:00 da manhã. As características particulares da operação das empresas, notadamente a possibilidade de realização de tarefas de manutenção durante o período noturno, permitem que restrições sejam eliminadas do modelo. Os resultados obtidos pelo autor permitem a solução do problema da empresa dia a dia, mas não resolve problemas de tarefas de manutenção, de famílias de aeronaves com tipos diferentes ou restrições de alocação de aeronaves específicas a certas rotas.

\section{MODELO MATEMÁTICO}

A modelagem proposta para o TAP neste trabalho é baseada em programação linear inteira. Considera um grafo $G=(N, A)$, onde $N$ é um conjunto de nós e $A$ é um conjunto de arcos; $K$ é o conjunto das aeronaves da subfrota candidata à realização de cada voo. Cada nó $n \in N$ representa um voo ou uma atividade de manutenção a ser realizado. Cada $\operatorname{arco} a(i, j) \in A$ representa uma conexão viável entre dois voos ou entre um voo e uma atividade de manutenção. A seguir é apresentada a notação utilizada nos modelos matemáticos deste trabalho.

\section{Conjuntos}

A conjunto de arcos que representam as conexões viáveis entre os nós da rede;

$K \quad$ conjunto das aeronaves candidatas a realizar os voos;

Mn cada subconjunto de nós que representam oportunidades de manutenção que ocorrem de forma simultânea em um mesmo aeroporto,

$N \quad$ conjunto dos nós da rede que representam voos ou oportunidades de manutenção.

\section{Variáveis de Decisão}

$x_{i j k} \quad$ tem valor 1 se o nó $i$ é conectado ao nó $j$ pela aeronave $k$; 0 caso contrário;

$Y k \quad$ tem valor 1 se a aeronave $k$ é utilizada na solução do problema; 0 caso contrário.

Destaca-se que existem apenas as variáveis de decisão $x_{i j k}$ que representam as conexões viáveis, ligando voos que terminam e começam no mesmo aeroporto e que respeitam o intervalo mínimo de conexão entre voos que chegam e voos que partem.

\section{Variáveis Auxiliares}

$\operatorname{DifHr}_{k}^{+}$quantidade de horas de voo acima do ideal atribuídas à aeronave $k$;

$\mathrm{DifHr}_{k}^{-}$quantidade de horas de voo abaixo do ideal atribuídas à aeronave $k$;

Dif $L d g_{k}^{+}$quantidade de pousos acima do ideal atribuídos à aeronave $k$;

$\operatorname{Dif} L d g_{k}^{-}$quantidade de pousos abaixo do ideal atribuídos à aeronave $k$;

$\mathrm{FCHr}_{k}$ custo fictício total relativo à diferença entre horas ideais e atribuídas à aeronave $k$;

$F C L d g_{k}$ custo fictício total relativo à diferença de pousos ideais e atribuídos à aeronave $k$. 
$O V B C_{i} \quad$ custo total de overbooking do voo $i$

$S P_{i}^{+} \quad$ quantidade de spoilage do voo $i$;

$S P_{i}^{-} \quad$ quantidade de overbooking do voo $i$;

$S P C_{i} \quad$ custo total de spoilage do voo $i$;

\section{Parâmetros}

$\mathrm{AvgHr}_{k}$ quantidade ideal de horas de voo que deve ser atribuída à aeronave $k$;

$A v g L d g_{k}$ quantidade ideal de pousos que deve ser atribuída à aeronave $k$;

$C A P_{k} \quad$ capacidade de assentos da aeronave $k$. 0 modelo não prevê a possibilidade de uma mesma aeronave ter capacidade reduzida em função de limitações de desempenho em um certo voo e/ou aeroporto. A alocação das aeronaves prevê capacidade constante de assentos para cada aeronave;

$\mathrm{CHr}_{k} \quad$ custo de uma hora de voo da aeronave $k$, excluindo o custo de combustível;

$C I_{i} \quad$ custo interno de conexão do nó $i$ quando coberto pela aeronave $k$. Esse custo aparece quando um nó representa mais de um voo. Isso ocorre quando é utilizada a estratégia de redução de nós, descrita na seção 5.5;

$c_{i j k} \quad$ custo da conexão do nó $i$ com o nó $j$ pela aeronave $k$. Inclui a taxa de pouso do nó $i$, quando realizado pela aeronave $k$;

$\mathrm{CO}_{i k} \quad$ custo do combustível do voo $i$ quando realizado pela aeronave $k$. Tem valor zero para os nós de atividades de manutenção;

$\mathrm{COVB}_{i} \quad$ custo de overbooking de um passageiro no voo $i$;

$C M_{i} \quad$ custo de manutenção do nó $i$. Tem valor zero para nós que representam voos;

$C S P_{i} \quad$ custo de spoilage de um passageiro no voo $i$

dem $_{i} \quad$ demanda de passageiros esperada para o voo $i$;

$d_{k} \quad$ degradação de desempenho da aeronave $k$;

$F C n x_{i j} \quad$ valor fictício atribuído a um passageiro que permanece a bordo da aeronave entre os voos $i$ e $j$ se ambos forem realizados pela mesma aeronave. Note-se que o valor atribuído à permanência de cada passageiro a bordo da aeronave durante uma conexão não é determinístico, isto é, deve ser usado um valor que traduza o benefício gerado para o passageiro e que esteja ajustado em relação aos demais custos envolvidos na função objetivo;

$H r_{i} \quad$ tempo do voo $i$ (em horas). Tem valor zero para os nós de atividades de manutenção;

$L d g_{i} \quad$ quantidade de pousos realizados no nó $i$. Tem valor zero para os nós de atividades de manutenção;

$L_{k} \quad$ custo fictício da utilização da aeronave $k$ na solução;

MaxHrk quantidade máxima de horas de voo permitidas para a aeronave $k$;

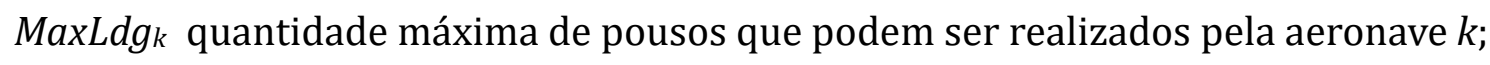

$O B_{j} \quad$ indica se o nó $j$ é obrigatório (valor 0 ) ou não obrigatório (valor 1);

$O V B C_{i} \quad$ custo de overbooking do voo $i$;

$P a x_{i j} \quad$ quantidade de passageiros em conexão entre os voos $i$ e $j$;

$\mathrm{PenHr}+$ custo fictício para cada hora de voo atribuída acima do ideal;

PenHr - custo fictício para cada hora de voo atribuída abaixo do ideal;

PenLdg ${ }^{+}$custo fictício para cada pouso atribuído acima do ideal;

PenLdg - custo fictício para cada pouso atribuído abaixo do ideal; 
$S P C_{i} \quad$ custo de spoilage do voo $i$.

O objetivo do modelo é assegurar a realização de todos os voos pelas aeronaves $k \in K$, com o menor custo possível. 0 modelo completo é apresentado a seguir:

Objetivo:

$$
\begin{aligned}
& \min \sum_{k \in K} \sum_{i \in N} \sum_{j \in N} c_{i j k} x_{i j k}+\sum_{k \in K} L_{K} y_{k}+\sum_{k \in K} \sum_{i \in N} \sum_{j \in N} H_{i} C H r_{k} x_{i j k}+ \\
& \sum_{k \in K} \sum_{i \in N} \sum_{j \in N} C_{i} x_{i j k}+\sum_{k \in K} \sum_{i \in N} \sum_{j \in N} C_{i k} d_{k} x_{i j k}+ \\
& \sum_{k \in K} \sum_{i \in N} \sum_{j \in N} C M_{i} x_{i j k}+ \\
& \sum_{i \in N} O V B C_{i}+\sum_{i \in N} S P C_{i}+\sum_{k \in K} \text { FairCostHr }_{k}+\sum_{k \in K} \text { FairCostLdg }_{k}- \\
& \sum_{k \in K} \sum_{i \in N} \sum_{j \in N} \text { Pax }_{i j} \text { FCnx }_{i j} x_{i j k}
\end{aligned}
$$

Sujeito a:

$$
\begin{aligned}
& \left(\sum_{i \in N} \sum_{k \in K} x_{i j k}\right)+O B_{j} \geq 1 \quad \forall j \in N \\
& \sum_{j \in N>i \in N} \sum_{k \in K} x_{i j k} \leq 1 \quad \forall i \in N \\
& \sum_{j \in N} x_{i j k}-\sum_{j \in N} x_{j i k}=0 \quad \forall i \in N, k \in K \\
& x_{1 j k} \leq y_{k} \quad \forall k \in K \\
& \sum_{i \in N} \sum_{j \in N} x_{i j k} H r_{i} \leq \operatorname{MaxHr}_{k} \quad \forall k \in K
\end{aligned}
$$

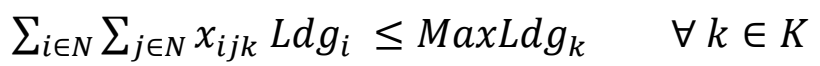

$$
\begin{aligned}
& \left(\sum_{j \in N} \sum_{k \in K} C A P_{k} x_{i j k}\right)-d_{e m}=S P_{i}^{+}-S P_{i}^{-} \quad \forall i \in N \\
& S P C_{i}=S P_{i}^{+} \operatorname{CSP}_{i} \quad \forall i \in N \\
& O V B C_{i}=S P_{i}^{-} \operatorname{COVB}_{i} \quad \forall i \in N \\
& \left(\sum_{i \in N} \sum_{j \in N} x_{i j k} H r_{i}\right)-A v g H r_{k}=D_{i f H r_{k}^{+}}-\operatorname{DifHr}_{k}^{-} \quad \forall k \in K \\
& \text { FairCostHr }_{k}=\text { DifHr }_{k}^{+} \text {PenHr }^{+}+\text {DifHr }_{k}^{-} \text {PenHr }^{-} \forall k \in K
\end{aligned}
$$

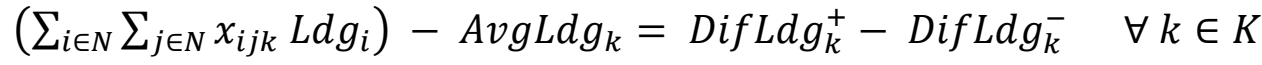

$$
\begin{aligned}
& \text { FairCost } L d g_{k}=\operatorname{DifLdg}_{k}^{+} \text {PenLdg }^{+}+\operatorname{DifLdg}_{k}^{-} \text {PenLdg }^{-} \forall k \in K \\
& S P_{i}^{+}, S P_{i}^{-}, \text {DifHr } r_{k}^{+}, \text {DifHr } r_{k}^{-}, \text {DifLdg } g_{k}^{+}, \text {Dif } L d g_{k}^{-} \geq 0 \\
& x_{i j k}, y_{k} \in\{0,1\}
\end{aligned}
$$

As restrições podem ser descritas do seguinte modo:

- (2) garante que todos os nós obrigatórios sejam cobertos;

- (3) garante que cada voo seja coberto apenas uma vez;

- (4) garante a continuidade da rede;

- (5) ativa a variável $y_{k}$ para as aeronaves utilizadas na solução do problema;

- (6) garante que cada aeronave não exceda a quantidade máxima de horas de voo permitida;

- (7) garante que cada aeronave não exceda a quantidade máxima de pousos permitidos;

- (8) determina a quantidade de passageiros em overbooking e a quantidade de spoilage de cada voo; 
- (9) determina o custo de spoilage de cada voo;

- (10) determina o custo de overbooking de cada voo;

- (11) determina a quantidade de horas de voo acima ou abaixo do ideal realizadas por cada aeronave;

- (12) determina o custo fictício relativo a diferença entre as horas de voo atribuídas e as horas de voo ideais de cada aeronave;

- (13) determina a quantidade de pousos acima ou abaixo do ideal realizados por cada aeronave;

- (14) determina o custo fictício relativo a diferença entre os pousos atribuídos e ideais de cada aeronave;

- (15) garante a consistência das variáveis irrestritas em sinal;

- (16) define o espaço das variáveis de decisão.

\subsection{Agrupamento de Nós}

As possibilidades de alocação de diferentes aeronaves em um dado voo ocorrem principalmente nos hubs das empresas, onde há diversas aeronaves no solo simultaneamente. Já nas extremidades opostas de cada um dos spokes, analisando-se o período de solução do TAP, é possível identificar momentos em que não ocorre simultaneidade de operação de aeronaves da empresa em determinados aeroportos, isto é, apenas uma aeronave da empresa encontra-se pousada de cada vez em certos aeroportos. Quando isso acontece, a conexão entre o voo de chegada e o de saída é obrigatória, pois não existe outra opção de aeronave para realizar o voo que parte a não ser aquela que chegou minutos antes. Nestes casos, é possível unificar os nós de chegada e de saída em um único nó. Essa simplificação do modelo não traz prejuízos ao resultado da otimização e possibilita a redução da quantidade de variáveis de decisão e, consequentemente, do tempo de processamento.

\section{MÉTODO HEURÍSTICO}

Como alternativa ao modelo matemático exato, foi desenvolvido um método heurístico para a solução do TAP, com vistas a obter soluções de custo próximo ao do modelo exato, porém, em tempos computacionais menores. 0 método heurístico apresentado divide-se em três partes: heurística construtiva, heurística de melhoria por trocas de trilhos e heurística de melhoria por trocas de voos.

A heurística construtiva é do tipo gulosa, pois trata-se de um método que ordena os voos em ordem crescente de horário e aloca cada um à próxima aeronave disponível no aeroporto de origem para criar uma solução inicial. Neste processo, não há a preocupação com a criação de soluções de boa qualidade, mas sim na criação rápida de uma solução viável que será utilizada como ponto de partida para as heurísticas de melhoria. Uma vez que este trabalho propõe solucionar o TAP tanto para um único tipo de aeronave, como para famílias com mais de um modelo, a heurística construtiva busca inicialmente alocar os voos a aeronaves do tipo originalmente planejado para cada voo e, caso seja uma solução para famílias, num segundo momento, aloca os voos restantes a qualquer aeronave disponível, independentemente de seu tipo. Essa segunda visita às aeronaves disponíveis visa a não deixar voos descobertos na solução inicial, o que caracterizaria uma solução inviável, já que o problema deve garantir a cobertura de todos os voos. A Figura 1 mostra o pseudocódigo da heurística construtiva. 
Uma vez executada a heurística construtiva, cada aeronave passa a ter um trilho de voos e o custo da solução heurística é calculado da mesma forma que a função objetivo do modelo exato. A primeira heurística de melhoria realiza trocas entre trilhos completos de duas aeronaves sorteadas aleatoriamente. Essa heurística é baseada no método 2-OPT (Johnson e McGeosh, 1997). Caso uma solução melhor (de menor custo) seja encontrada, tal solução é preservada e a anterior, descartada. 0 processo é repetido até que não seja encontrada uma solução melhor após a quantidade de tentativas definida para execução da heurística. A Figura 2 apresenta o pseudocódigo da heurística de troca de trilhos.

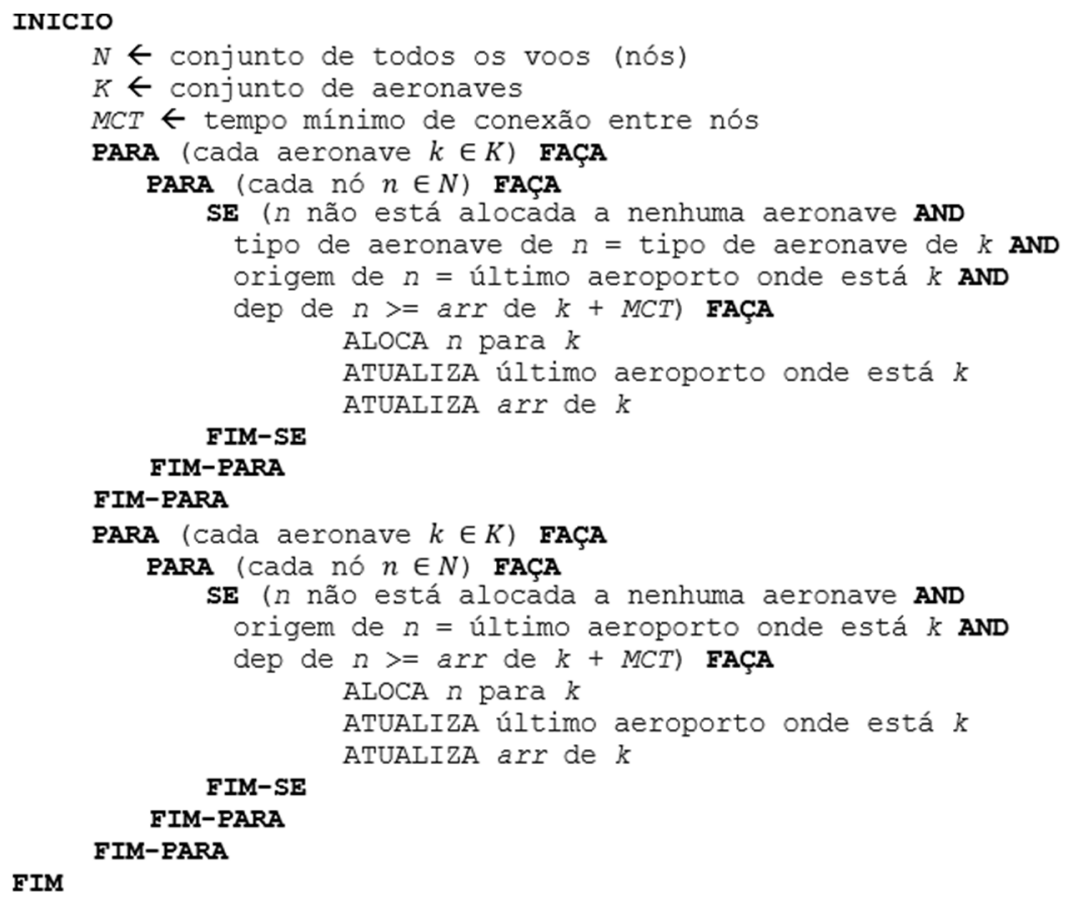

Figura 1. Heurística construtiva

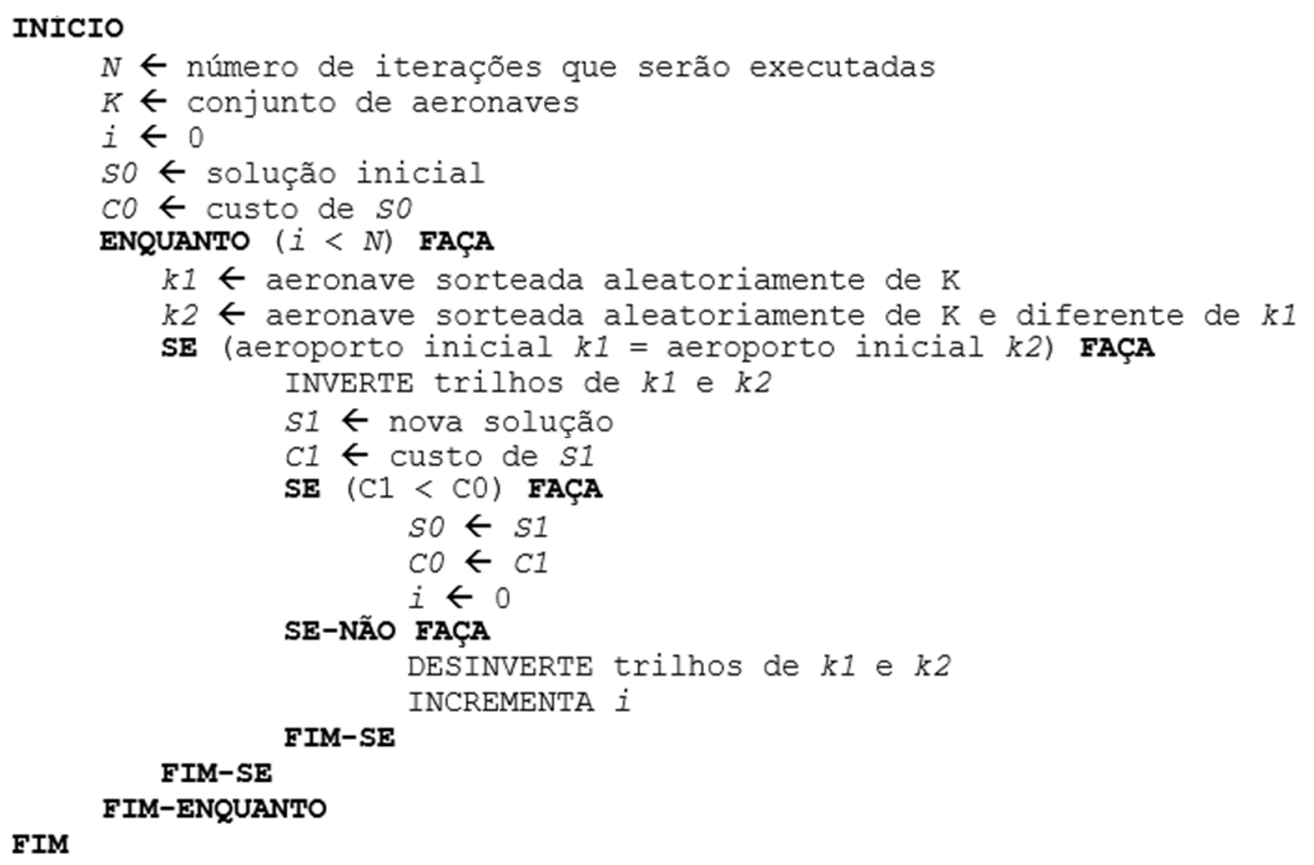

Figura 2. Heurística de melhoria por troca de trilhos 
Após a aplicação da heurística de melhoria inicial, realizando a troca de trilhos, uma segunda heurística de melhoria é aplicada, realizando a troca de voos ou parte de trilhos entre aeronaves sorteadas aleatoriamente. Para evitar tentativas de trocas repetitivas com aeronaves já utilizadas nas trocas, a heurística implementa uma lista tabu, isto é, uma lista de aeronaves que ficam impedidas de serem utilizadas por um certo número de iterações. A implementação da lista tabu baseia-se na implementação apresentada por Glover (1989). 0 tamanho da lista tabu significa quantas aeronaves já sorteadas em iterações anteriores da heurística de troca de voos ficam impedidas de serem utilizadas novamente nas iterações seguintes. Tal impedimento tem por objetivo aumentar a diversidade de aeronaves sorteadas para as tentativas de trocas de voos e, consequentemente, gerar diferentes combinações e possíveis melhorias da solução. A Figura 3 apresenta o pseudocódigo da heurística de troca de voos.

\section{TESTES E APLICAÇÕES}

Para a realização de testes dos modelos, foram utilizadas instâncias reais, obtidas de uma empresa aérea brasileira. A empresa opera uma frota de 35 aeronaves da mesma família, mas de três tipos diferentes. Foram utilizadas instâncias de tamanhos diferentes, a saber: problemas de alocação dos voos de apenas um dia a aeronaves de um tipo, tipicamente encontrados no cenário operacional da empresa, onde realocações de voo para um período pequeno precisam ser feitas após alguma ocorrência (problemas técnicos, meteorológicos, etc.); problemas de alocação de voos de períodos de sete dias, normalmente encontrados no planejamento tático da empresa, onde é realizada a alocação dos voos da semana subsequente; e problemas de períodos de dez ou quatorze dias, resolvidos para fins de planejamento estratégico e definição de viabilidade de execução de uma malha proposta pela frota da empresa.

As instâncias foram nomeadas de acordo com um padrão, de forma a serem facilmente identificadas pelo nome. Os quatro primeiros caracteres indicam o tipo de aeronave planejado, os dois caracteres seguintes indicam a quantidade de aeronaves utilizada, os dois caracteres seguintes indicam a quantidade de dias a que se refere a malha utilizada para gerar a instância. 0 código A32F refere-se à frota composta por aeronaves A318, A319 e A320. Exemplo: A318_06_07 significa um problema da frota de aeronaves tipo A318, com seis aeronaves e voos em sete dias.

Os testes de execução foram realizados em um microcomputador PC Intel Core i7 3770k, 3.5GHz, com $16 \mathrm{~Gb}$ de memória RAM e sistema operacional Microsoft Windows 7 Professional. 0 modelo exato foi implementado utilizando linguagem Java, Runtime Enviroment versão 7.0 e o pacote de otimização Gurobi versão 6.0.0 (Gurobi, 2015), sem alteração dos parâmetros padrão de execução. 0 método heurístico também foi implementado em linguagem Java. Cada uma das instâncias foi executada dez vezes em cada método (exato e heurístico), a fim de eliminar distorções de tempo de processamento computacional. Os parâmetros medidos foram o custo da solução (valor da função objetivo) e tempo de processamento para geração da solução com cada um dos métodos.

O algoritmo de agrupamento de nós foi testado com vistas a determinar sua efetividade na redução do tempo de processamento, bem como no possível aumento do custo da solução. Em todas as instâncias os valores do custo (função objetivo) com e sem a aplicação do algoritmo de redução de nós foi o mesmo, não havendo, portanto, prejuízo em sua utilização.

No método heurístico, quatro parâmetros precisam ser calibrados antes de sua utilização para a solução de instâncias do problema TAP: quantidade de iterações da heurística de troca 
de trilhos, quantidade de iterações da heurística de troca de voos e tamanho da lista tabu de aeronaves e quantidade de ciclos de melhoria. Para realizar esses ajustes, foram realizados testes com a instância A32F_35_02. Essa instância foi escolhida por ser um problema de tamanho considerável, envolver aeronaves de todos os tipos da família e usar a frota inteira da empresa.

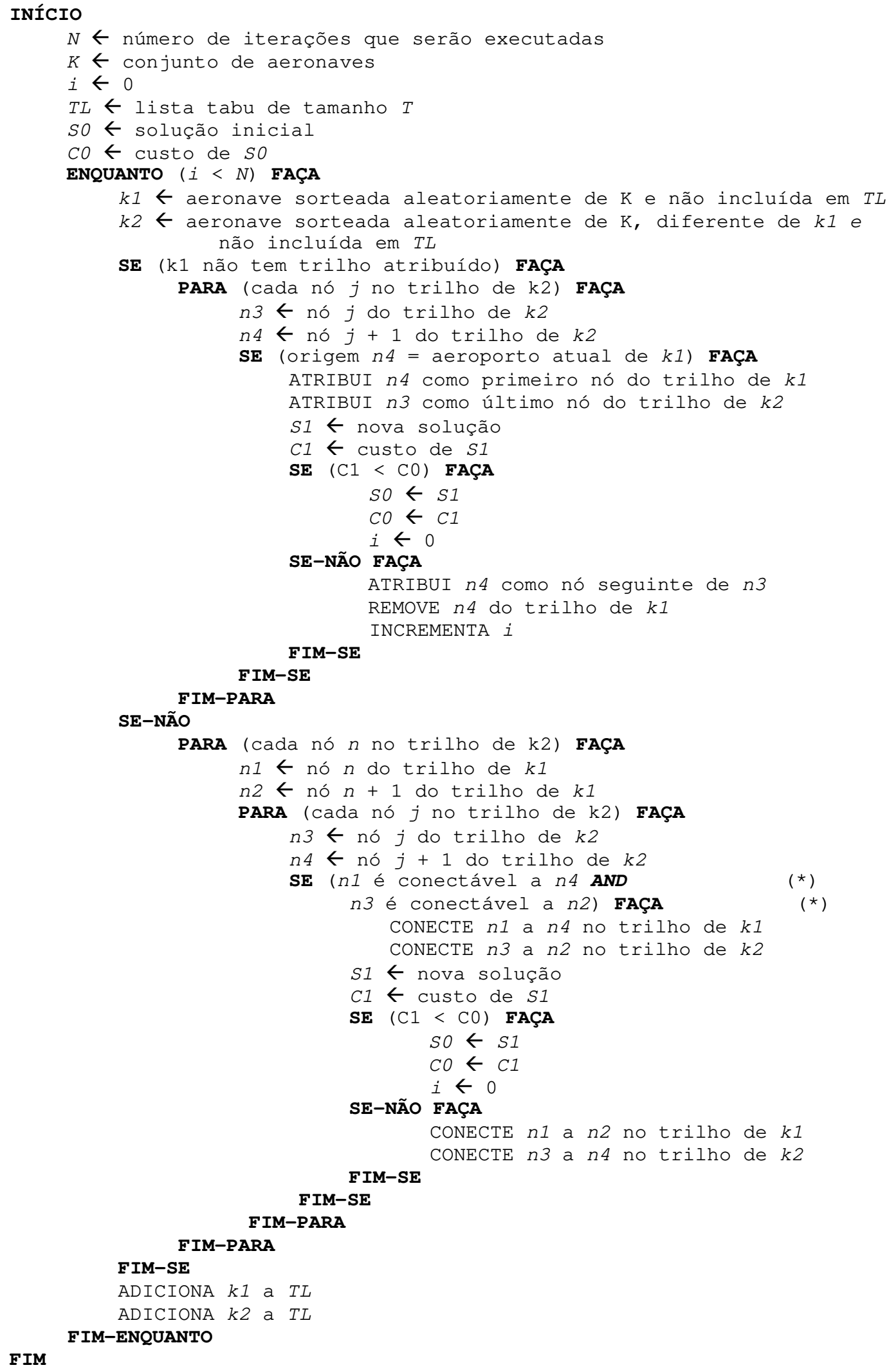

Figura 3. Heurística de melhoria por troca de voos 
Para a calibração da heurística de troca de trilhos, foi gerada uma solução inicial e, a partir dela, foi aplicada a heurística de troca de trilhos por um número variável de vezes, até que o critério de parada fosse atingido. Analisando-se os resultados obtidos, nota-se que o ganho obtido após o processamento da heurística de troca de trilhos é baixo, da ordem de 0,05\%, e que, a partir de 300 iterações o melhor valor obtido fica estável, havendo apenas diminuição do desvio padrão. Essa diminuição sugere que os valores médios, e consequentemente as diversas soluções obtidas, convergem com o aumento de iterações, até estabilizarem a partir de 800 iterações. Uma vez que o tempo de processamento varia pouco com o aumento de iterações, optouse por utilizar 1000 iterações da heurística de troca de trilhos para os testes com os problemas reais.

Para a calibração da heurística de troca de voos, foi utilizada a mesma solução inicial gerada para a calibração da heurística de troca de trilhos e aplicada a heurística de troca de voos por um número variável de vezes, até que o critério de parada fosse atingido. Os resultados obtidos mostram que a heurística de troca de voos possibilita ganhos da ordem de até 2,03\% em relação à solução inicial apresentada, porém, apresenta desvio padrão maior e sem convergência com o aumento de iterações. É possível notar também que o tempo de processamento é proporcional ao aumento de iterações. Com isso, optou-se por utilizar 6000 iterações sem melhoria como critério de parada para a heurística de troca de voos.

Uma vez realizadas as calibrações dos critérios de parada das heurísticas de troca de trilhos e troca de voos, passou-se à calibração do tamanho da lista tabu de aeronaves na heurística de troca de voos. Para tanto, variou-se o tamanho de lista tabu, desde zero (sem lista) até 33 aeronaves de um total de 35. A Figura 4 mostra a variação do tempo de processamento em função do tamanho da lista tabu, e a Figura 5 mostra os valores de custo médio e melhor custo obtido em função do tamanho da lista tabu e a Tabela 1 resume os resultados das aplicações realizadas.

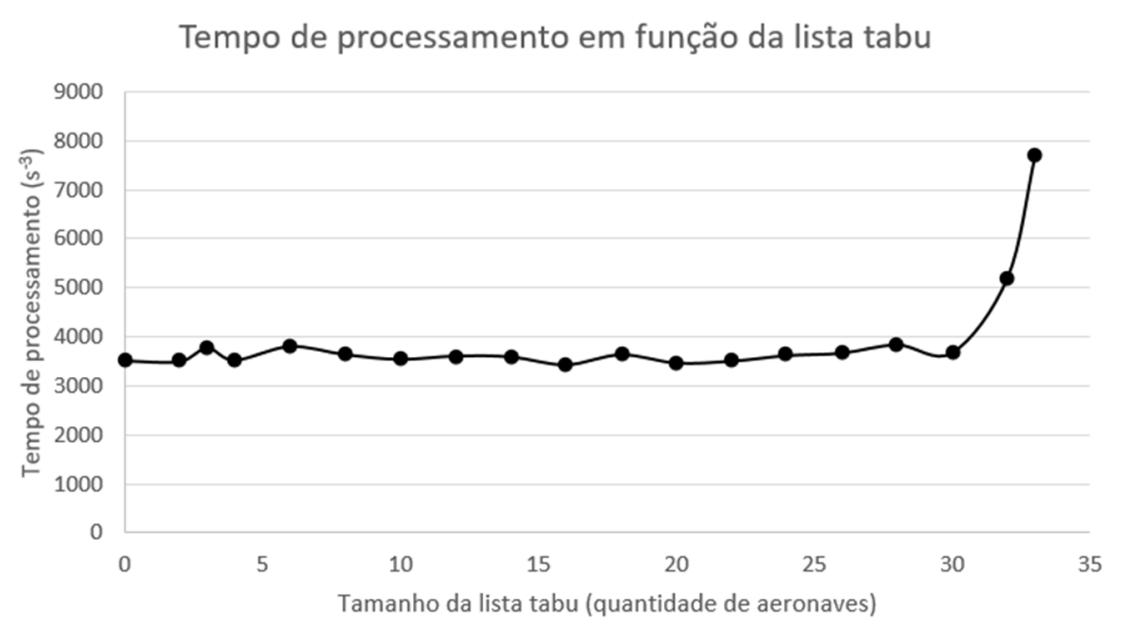

Figura 4. Tempo de processamento em função do tamanho da lista tabu

Analisando-se a variação do tempo de processamento em função do tamanho da lista tabu, percebe-se que a variação é pequena, exceto para tamanhos de lista que se aproximam da quantidade total de aeronaves, indicando que listas muito grandes não são adequadas para a solução do problema. Já a análise dos custos de solução obtidos mostra que há variações do custo médio inerentes à característica aleatória das soluções geradas, mas com uma melhor solução de valor 
praticamente constante para a maior parte dos testes. Com isso, optou-se por definir o tamanho da lista em $23 \%$ da quantidade de aeronaves do problema, sendo este um tamanho que proporciona uma diversidade suficiente na escolha de aeronaves para a realização das tentativas de melhoria, uma vez que a lista é dinâmica, mantendo sempre as últimas aeronaves sorteadas como impedidas, sem comprometer o tempo de execução do programa.

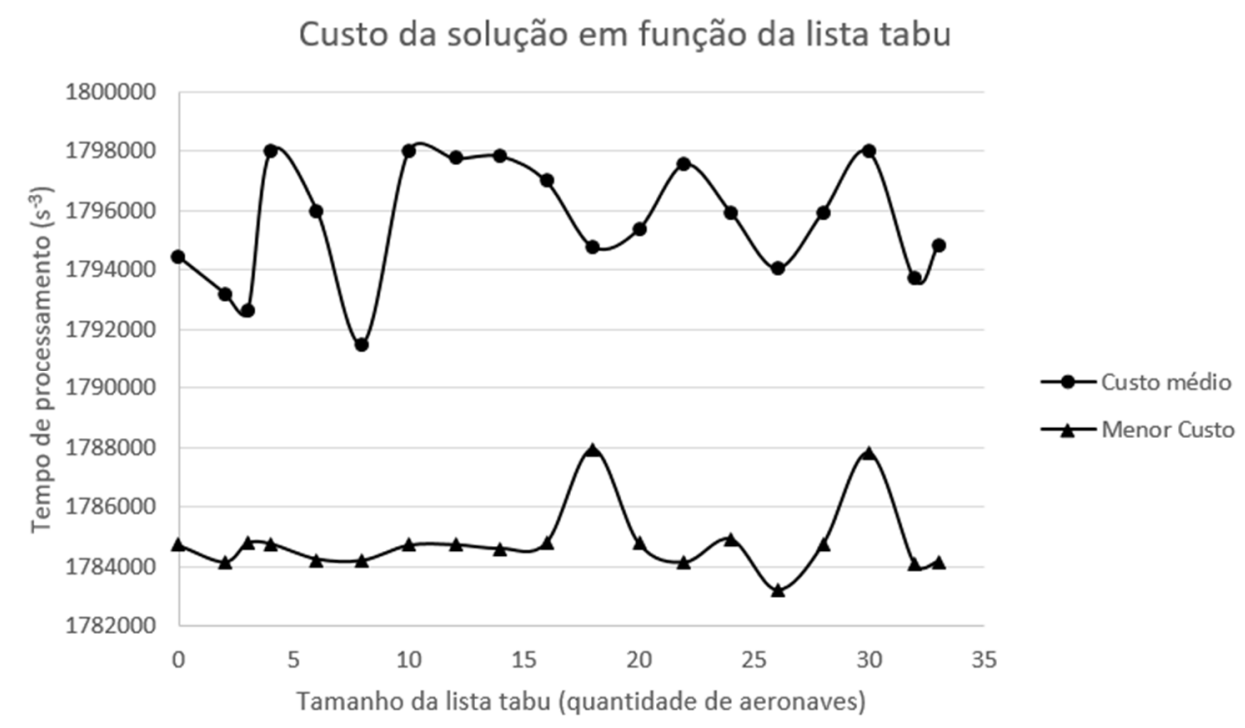

Figura 5. Custo da solução em função do tamanho da lista tabu

Tabela 1: Resultados para instâncias de problemas reais

\begin{tabular}{|c|c|c|c|c|c|c|c|c|c|}
\hline Instância & Nós & Variáveis & Restrições & $\begin{array}{l}\text { Tempo } \\
\text { exato (s) }\end{array}$ & $\begin{array}{l}\text { Tempo } \\
\text { heurística } \\
\text { (s) }\end{array}$ & Dif (\%) & $\begin{array}{l}\text { Valor médio } \\
\text { todo exato }\end{array}$ & $\begin{array}{l}\text { Valor médio } \\
\text { todo } \\
\text { heurístico }\end{array}$ & Dif (\%) \\
\hline A319_04_01 & 27 & 204 & 199 & 0.364 & 0.337 & -7.29023 & 120800 & 120801 & 0.000 \\
\hline A319_04_02 & 54 & 452 & 331 & 0.735 & 0.555 & -24.4181 & 243491 & 245660 & 0.891 \\
\hline A319_04_07 & 216 & 2372 & 835 & 15.636 & 10.987 & -29.7316 & 911034 & 931293 & 2.224 \\
\hline A318_06_07 & 218 & 4912 & 1487 & 7.483 & 4.072 & -45.5908 & 914480 & 914481 & 0.000 \\
\hline A320_26_02 & 296 & 62108 & 10287 & 5483.581 & 1.786 & -99.9674 & 1357482 & 1357483 & 0.000 \\
\hline A32F_35_02 & 410 & 107830 & 16189 & 8374.096 & 3.560 & -99.9575 & 1775896 & 1793688 & 1.002 \\
\hline A32F_10_07 & 432 & 21748 & 3828 & 348.037 & 13.421 & -96.1439 & 1564789 & 1564790 & 0.000 \\
\hline A319_04_14 & 432 & 125912 & 5239 & 152.271 & 73.113 & -51.9852 & 1817875 & 1827456 & 0.527 \\
\hline A320_26_03 & 442 & 137994 & 15271 & $55080(*)$ & 4.778 & -99.9913 & 2027765 & 2023590 & -0.206 \\
\hline A320_26_04 & 591 & 231558 & 19919 & & 13.071 & & & 2712247 & \\
\hline A32F_35_03 & 613 & 238042 & 24181 & & 9.465 & & & 2686651 & \\
\hline A320_26_05 & 745 & 361402 & 24959 & & 15.266 & & & 3425712 & \\
\hline A32F_35_04 & 829 & 408379 & 31803 & & 16.456 & & & 3659745 & \\
\hline A320_26_06 & 899 & 520284 & 29999 & & 24.480 & & & 4137273 & \\
\hline A32F_35_05 & 1046 & 648997 & 40091 & & 30.531 & & & 4605177 & \\
\hline A320_26_07 & 1053 & 708234 & 35039 & & 36.069 & & & 4849545 & \\
\hline A32F_35_06 & 1263 & 942500 & 48379 & & 52.522 & & & 5566443 & \\
\hline A32F_35_07 & 1480 & 1293683 & 56667 & & 89.504 & & & 6534958 & \\
\hline A32F_35_10 & 2110 & 1713080 & 70064 & & 268.202 & & & 9331374 & \\
\hline
\end{tabular}


Realizou-se também ajuste da quantidade de ciclos de melhoria que devem ser realizados. Foram realizados testes com 1 a 5 ciclos. Analisando-se a variação do tempo de processamento em função da quantidade de ciclos, nota-se que o aumento de tempo é consistente com o aumento de ciclos. A variação no resultado é pequena, não havendo ganhos significativos com o aumento do número de ciclos. Com isso, optou-se por utilizar três ciclos das heurísticas de melhoria a cada execução do programa.

Após a validação e calibração dos modelos exato e heurístico, foram desenvolvidas instâncias de problemas reais baseadas na malha de uma empresa aérea brasileira que opera três tipos de aeronaves de uma mesma família. Foram criadas instâncias variando-se a quantidade de aeronaves de 4 até 35 e a quantidade de dias de 1 até 14 . A Tabela 1 apresenta as instâncias de problemas reais que foram resolvidas neste trabalho e a comparação dos resultados obtidos.

A instância A320_26_03 teve seu processamento pelo método exato interrompido após decorridos 55080 segundos. Tal interrupção justifica-se pelo fato de que no ambiente operacional das empresas aéreas, as decisões precisam ser tomadas rapidamente e, então, modelos que demoram muitas horas ou dias para rodar não são apropriados para suportar as tomadas de decisão pretendidas.

As instâncias que não apresentam tempo de processamento pelo método exato, não tiveram resultado obtido por esse método, tendo sido acusado erro de falta de memória durante o processamento.

\section{CONCLUSÕES E CONTINUIDADE}

Analisando os resultados das instâncias, é possível concluir que o modelo matemático funciona adequadamente, gerando soluções viáveis do TAP, bem como respondendo adequadamente a variações dos parâmetros de entrada, como custo de conexões entre voos, capacidade das aeronaves, conexões de passageiros, etc. Pode-se concluir também que, assim como esperado, o modelo matemático exato tem tempo de resposta exponencial em função da quantidade de nós do problema, o que comprova sua natureza NP-hard. Isto torna inviável a sua utilização para resolver problemas reais de empresas aéreas com frotas médias ou grandes, ainda que por períodos de tempos pequenos. Já a análise dos resultados obtidos pelos métodos exato e heurístico para os mesmos problemas, permite concluir que o método heurístico é rápido e fornece resultados cerca de $0,5 \%$ a 2,2\% abaixo do valor ótimo, mas em tempos computacionais adequados ao cenário operacional das empresas, que exige respostas rápidas.

Foram solucionados problemas de até 14 dias para subfrotas pequenas e até 10 dias para a frota completa de uma empresa de médio porte, o que mostra que o modelo heurístico pode ser utilizado no ambiente operacional de empresas aéreas.

Diante dos resultados obtidos, pode-se concluir que a principal contribuição deste trabalho é a utilização em conjunto de todas as restrições que devem ser consideradas por uma empresa aérea no processo de alocação de aeronaves a voos, permitindo que os modelos desenvolvidos sejam utilizados em operações comerciais, nos cenários estratégico, tático e operacional. As pequenas diferenças encontradas entre os resultados do modelo heurístico e do modelo exato, permitem concluir que o modelo heurístico produz soluções de boa qualidade em tempos computacionais adequados, apontando avanços na solução deste tipo de problema.

A partir dos resultados obtidos, é possível determinar quais os fatores que mais causam impacto no tempo de processamento do modelo exato. Calculando-se a correlação entre o tempo de processamento do modelo exato e as quantidades de aeronaves, restrições, variáveis e nós, 
obtém-se os seguintes resultados: $98,2 \%, 95,6 \%, 64,7 \%$ e $52,3 \%$, respectivamente. Isso indica que a quantidade de aeronaves é o principal fator determinante na complexidade das instâncias do problema e, consequentemente, no tempo necessário para a solução com o modelo exato. Tais correlações explicam grandes variações de tempo de processamento entre problemas com quantidades próximas de nós, como por exemplo as instâncias A32F_35_02 (410 nós) e A32F_10_07 (432 nós).

Já no caso do método heurístico, calculando-se a correlação entre o tempo de processamento e as quantidades de nós e aeronaves, os resultados são $81,2 \%$ e $29,4 \%$, respectivamente, mostrando que, neste caso, a quantidade de nós tem impacto mais significativo no tempo de processamento. Tal impacto deve-se à implementação do método heurístico, que faz sucessivas varreduras na lista de nós atribuídos às aeronaves a cada iteração, em busca de melhorias.

\subsection{Continuidade}

Entre as várias possibilidades que se apresentam para continuidade e melhoria deste trabalho, destacam-se:

- A execução de testes do modelo exato em computadores com memória RAM maior que $16 \mathrm{~Gb}$ e/ou executando máquinas virtuais Java especialmente configuradas com vistas à redução dos tempos de processamento, permitindo a solução de instâncias maiores;

- Apesar dos problemas típicos das empresas aéreas serem de 1 ou 7 dias e terem sido resolvidos problemas de até 10 dias, a solução de instâncias de problemas maiores pelo método heurístico, de até 30 dias por exemplo, a fim de verificar as limitações do método e possivelmente obter soluções de problemas de planejamento estratégico de longo prazo;

- A inclusão da limitação de capacidade de assentos de uma determinada aeronave em um determinado voo, substituindo-se o parâmetro $C A P_{k}$ por $C A P_{i k}$, que passaria a representar a capacidade da aeronave $k$ quando realizando o voo $i$;

- A possibilidade de utilizar as soluções heurísticas, utilizando-as como entrada do método exato. Espera-se, com isso, a obtenção de soluções ótimas para problemas maiores, mas com tempos computacionais viáveis;

- O desenvolvimento de um modelo integrado de solução dos problemas de geração e programação de voos, alocação de frotas e atribuição de aeronaves, com vistas a obter soluções de ótimo global para esses problemas;

- O desenvolvimento de um modelo integrado para solucionar os problemas de atribuição de aeronaves e de alocação de tripulantes de forma conjunta, de forma a minimizar as trocas de aeronaves realizadas pelos tripulantes durante suas programações de voo e para que, especialmente nos cenários de recuperação de malha, as soluções encontradas para alocação das aeronaves não sejam inviabilizadas pela falta de tripulações disponíveis nas bases da empresa.

\section{REFERÊNCIAS}

AIRBUS - Societé par Actions Simplifiée (2002) Getting to grips with aircraft performance monitoring. Toulouse, France.

AIRBUS - Societé par Actions Simplifiée (2005) Getting to grips with MMEL and MEL. Toulouse, France.

Ackert, S (2011). Engine Maintenance Concepts for Financiers. Elements of Turbofan Shop Maintenance Costs. Disponível em http://www.aircraftmonitor.com/uploads/1/5/9/9/15993320/engine_mx_concepts_for_financiers__v2.pdf (Acesso em 12/02/2016).

Alessandri, A.; A. Di Febbraro; A. Ferrara e E. Punta (1998) Optimal control of freeways via speed signaling and ramp metering. Control Engineering Practice, v. 6, n. 6, p. 771-780. DOI: 10.1016/S0967-0661(98)00083-5 
Bazargan, M. (2010) Airline Operations and Scheduling. Second Edition. p. 1-284.

Belobaba P.; A. Odoni e C. Barnhart (2009) The Global Airline Industry. John Willey \& Sons: West Sussex, UK.

Boeing Commercial Airplanes (2015) About the 737 Family. Disponível em http://www.boeing.com /boeing/commercial/737family/background.page (Acesso em 03/03/2015).

Caetano, D. J. (2011) Modelagem integrada para a programação de voos e alocação de frotas: abordagens baseadas em programação linear inteira e na meta-heurística Colônia de Formigas. Tese (Doutorado em Engenharia de Transportes), Escola Politécnica, Universidade de São Paulo, São Paulo.

Caetano, D. J. e N. D. Gualda (2015) F. Solving the Integrated Schedule Generation and Fleet Assignment Problem: an ACOBased Metaheuristic Approach. Journal of Transport Literature, v.9, n.3, p. 30-34. D0I: 10.1590/2238-1031.jtl.v9n3a6

Caetano, D. J. e N. D. F. Gualda (2011) Um modelo integrado para a programação de voos e alocação de frotas. Transportes (Rio de Janeiro), v.19, n.2, p. 16. DOI: 10.14295/transportes.v19i2.209

Clarke, L. et al. (1997) The aircraft rotation problem. Annals of Operations Research. v. 69, p. 33-46. DOI: 10.1023/A:1018945415148

DECEA - Departamento de Controle do Espaço Aéreo (s/d) Tarifas de Navegação Aérea - Regras Gerais de Cobrança. Disponível em: http://tarifas.decea.gov.br/regras.aspx (Acesso em 01/03/2015)

Fregnani, J. A. T. G. (2007) Um modelo para minimização dos custos totais de abastecimento considerando as múltiplas escalas das aeronaves nas rotas de uma empresa aérea brasileira. Dissertação (Mestrado em Engenharia de Infraestrutura Aeronáutica), Instituto Tecnológico da Aeronáutica, São José dos Campos.

Glover, F. (1989) Tabu Search - Part I. ORSA Journal of Computing, v. 1, n. 3, p. 190-206. DOI: 10.1287/ijoc.1.3.190

Gomes, W. P. e N. D. F. Gualda (2011) Modelagem Integrada do Problema de Programação de Tripulantes de Aeronaves. Transportes (Rio de Janeiro), v. 19, n.1, p. 23-32.

Gomes, W. P. e N. D. F. Gualda (2015) Heuristics to solve the integrated airline crew assignment problem. Journal of Transport Literature. v. 9, n. 1, p. 25-29. Disponível em: https://dx.doi.org/10.1590/2238-1031.jtl.v9n1a5

González, J. J. S. (2014) Approaches to solve the fleet-assigment, aircraft-routing, crew-pairing and crew-rostering problems of a regional carrier. Omega The International Journal of Management Science, n. 43, p. 71-82. DOI: 10.1016/j.omega.2013.06.006

Grönkvist, M. (2005) The Tail Assignment Problem. PhD Dissertation (Department of Computer Science and Engineering), Chalmers and Göteborg University, Gothenburg, Swedish.

GRU Airport (2014) Tabela de Tarifas do GRU Airport - Aeroporto Internacional de São Paulo. Disponível em http://www.gru.com.br/pt-br/Tarifas (Acesso em 26/10/2014)

GUROBI (2015) Gurobi Optimizer. State of Art Mathematical Programming Solver. Disponível em http://www.gurobi.com/products/gurobi-optimizer/gurobi-overview (01/03/2015).

INFRAERO - Empresa Brasileira de Infra-Estrutura Aeroportuária (2014) Tarifário - Tarifas Aeroportuárias e de Navegação Aérea, Brasília.

INFRAMÉRICA (2014) Tarifas. Disponível em http://www.bsb.aero/br/tarifas/ (Acesso em 26/10/2014)

Jarrah, A. I. e J. C. Strehler (2000) An optimization model for assigning through flights. IEEE Transactions, v.32, n.3, p. 237-244.

Johnson, D. S. e L. A. Mcgeosh (1997) The Traveling Salesman Problem: A Case Study in Local Optimization. In: Aarts, E. H. L. e Lenstra, J. K. (eds.) Local Search in Combinatorial Optimization. John Willey and Sons: London, UK,.

Klabjan, D. (2004) Large-scale models in the airline industry. In G. Desaulniers, J. Desroriers, M. M. Solomon (eds.) Column Generation. Kluwer Academic Publishers.

Lapp, M. e F. Wikenhauser (2012) Incorporating aircraft efficiency measures into the tail assignment problem. Journal of Air Transport Management,. n. 19, p. 25-30.

Martin, T. W. (2011) Airlines Brace for Increases in Jet-Fuel Prices. Disponível em http://online.wsj.com/news/articles/SB10001424052748703905404576164741506652956 (Acesso em 13/06/2014)

Mcafee, R. P. e V. T. Velde (2007) Dynamic Pricing in the Airline Industry. In: Hendershott, T. J. (eds.) Handbook on Economics and Information Systems. Elsevier Handbooks in Information Systems, v. 1. ISBN 0444517715, 2007

Mc Cartney, S. (2012) How Airlines Spend Your Airfare. Disponível em http://online.wsj.com/news/articles /SB10001424052702303296604577450581396602106 (Acesso em 13/06/2014)

Simpson, R. W. e P. P. Belobaba (1992) The Demand for Air Transportation Services. Notes for Air Transportation Economics Course 16.74. Massachusetts Institute of Technology.

Sriram, C. e A. Haghani (2003) An optimization model for aircraft maintenance scheduling and re-assignment. Transportation Research Part A. n. 37, p. 29-48.

Subramanian, R. et al. (1994) Coldstart: Fleet Assignment at Delta Airlines. Interfaces, v. 24, n. 1, p. 104-120. 2002. 